\title{
ClpXP-Dependent RpoS Degradation Enables Full Activation of Type III Secretion System, Amylovoran Production, and Motility in Erwinia amylovora
}

\author{
Jae Hoon Lee and Youfu Zhao ${ }^{\dagger}$
}

Department of Crop Sciences, University of Illinois at Urbana-Champaign, Urbana 61801. Accepted for publication 7 July 2017.

ABSTRACT

\begin{abstract}
Erwinia amylovora, the causal agent of fire blight disease of apple and pear, employs intracellular proteases, including Lon and ClpXP, for posttranslational regulation of various cellular proteins. It has been shown that Lon plays a critical role in E. amylovora virulence by directly targeting type III secretion system (T3SS) proteins and the Rcs phosphorelay system. In this study, we genetically examined the role of ClpXP and its potential interaction with Lon in E. amylovora. Mutation in clpXP diminished the expression of the T3SS, reduced exopolysaccharide amylovoran production and motility, and resulted in delayed disease progress. Western blot analyses showed highly accumulated RpoS proteins in the clpXP
\end{abstract}

mutant. Moreover, mutation of rpoS in the $\operatorname{clpXP}$ mutant background rescued the expression of the T3SS and amylovoran production, suggesting that ClpXP-dependent RpoS degradation positively affects virulence traits. Interestingly, lack of both ClpXP and Lon resulted in significantly reduced virulence but increased expression of the T3SS and amylovoran production. However, this phenomenon was independent of RpoS accumulation, suggesting that ClpXP and Lon are indispensable for full virulence in E. amylovora.

Additional keywords: $\sigma$ factor.
Fire blight disease, caused by an enterobacterium Erwinia amylovora, is one of the most economically important diseases in the plant family Rosaceae. It has been demonstrated that E. amylovora utilizes two major virulence factors: the hypersensitive response and pathogenicity ( $h r p$ )-type III secretion system (T3SS) and the exopolysaccharide (EPS) amylovoran (Khan et al. 2012; Oh and Beer 2005). The hrp-T3SS directly injects virulenceassociated proteins into host cells to overcome host defense response and promote disease development (Nissinen et al. 2007). The expression of $h r p$-T3SS genes is activated by the master regulator HrpL, a member of the extracytoplasmic functions subfamily of $\sigma$ factors (McNally et al. 2012; Wei and Beer 1995). Recent studies showed that the alternative $\sigma$ factor $54(\mathrm{RpoN})$, its modulation protein $\mathrm{YhbH}$, enhancer binding protein $\mathrm{HrpS}$, and the nucleoid-associated protein IHF are all essential for the expression of $h r p L$ and other $h r p$-T3SS genes (Ancona et al. 2014; Lee and Zhao 2016; Lee et al. 2016). Moreover, the RpoN-HrpL alternative $\sigma$ factor cascade is further activated by the bacterial alarmone (p)ppGppmediated stringent response under nutrient-limited conditions (Ancona et al. 2015). On the other hand, the EPS amylovoran is a heteropolymer composed of pentasaccharide-repeating units and contributes to pathogenesis through biofilm formation and vessel blockage in plants (Koczan et al. 2009; Nimtz et al. 1996). Amylovoran biosynthesis genes are encoded by the 12-gene ams operon, which is positively regulated by the Rcs phosphorelay system (Bernhard et al. 1993; Wang et al. 2009, 2012).

ClpXP and Lon are two major proteases in bacteria, belonging to the $\mathrm{AAA}^{+}$(ATPase-associated with diverse cellular activities) family proteins, and control the abundance and quality of intracellular proteins in response to a wide variety of environments (Sauer and

†Corresponding author: Y. Zhao; E-mail: zhao888@illinois.edu

*The $\boldsymbol{e}$-Xtra logo stands for "electronic extra" and indicates that one supplementary table and two supplementary figures are published online.

(c) 2017 The American Phytopathological Society
Baker 2011). Previous studies have indicated that proteolytic activities from ClpXP and Lon are required during bacterial pathogenesis (Bretz et al. 2002; Iyoda and Watanabe 2005; Losada and Hutcheson 2005; Yamamoto et al. 2001). It was recently reported that Lon plays a major role in regulation of virulence in E. amylovora by directly targeting HrpS and HrpAT3SS proteins, as well as RcsA, an auxiliary response regulator of the Rcs phosphorelay system (Lee et al. in press). Furthermore, accumulation of RcsA/RcsB in the lon mutant led to upregulation of $h r p S$ and $a m s G$ and downregulation of $f l h D$ and small RNA $\operatorname{csr} B$. Suppression of $c s r B$ expression indirectly enhanced the activity of the RNA-binding carbon storage regulator A (CsrA) protein, which plays a central role in E. amylovora virulence (Ancona et al. 2016). However, the role of ClpXP in E. amylovora virulence remains unknown.

ClpXP is an ATP-dependent cytosolic protease, composed of $\mathrm{AAA}^{+}$ATPase $(\mathrm{ClpX})$ and proteolytic chamber (ClpP). ClpP alone can degrade damaged or misfolded peptides but its proteolytic activity becomes selective and tightly controlled only after association with ClpX (Frees et al. 2013). A number of proteins involved in diverse cellular processes, including metabolic enzymes and transcription factors, have been identified as ClpXP substrates (Flynn et al. 2003). Most importantly, ClpXP-mediated proteome changes are largely achieved by regulating RpoS activity (Schweder et al. 1996). RpoS is an alternative $\sigma$ factor induced during stationary phase and under stress conditions, and plays major roles in stress response and virulence regulation by altering stress sensitivity and virulence-associated phenotypes (Badger and Miller 1995; Fang et al. 1992; Flavier et al. 1998; Solis et al. 2006). A recent study showed that the rpoS mutant of E. amylovora exhibited increased sensitivity to oxidative, osmotic, acid, and heat stresses (Santander et al. 2014). On the other hand, RpoS activity is tightly controlled by multiple layers of regulatory elements, including ClpXP-dependent degradation, during favorable growth conditions (Battesti et al. 2011). The rate-limiting factor for RpoS degradation is the adaptor protein RssB, which facilitates RpoS recognition by ClpXP (Becker et al. 1999; Pruteanu and Hengge-Aronis 2002). Under stress conditions or during stationary phase, RssB inhibitors and low ATP 
levels release RpoS from degradation by ClpXP (Bougdour et al. 2006, 2008; Peterson et al. 2012). This process leads to increased RpoS levels and allows cells to activate genes involved in cell survival. A defect in controlling RpoS level has been reported to cause reduced virulence in enterohemorrhagic Escherichia coli (EHEC) and Dickeya dadantii (Iyoda and Watanabe 2005; Li et al. 2010).

We aimed to examine the role of ClpXP in Erwinia amylovora virulence and its potential interaction with Lon. Our results showed that RpoS accumulation in the $\operatorname{clpXP}$ mutants contributed to the suppression of T3SS, amylovoran production, and motility. Deletion of both $c l p X P$ and lon genes led to significantly reduced virulence independent of RpoS level and other virulence factors.

\section{MATERIALS AND METHODS}

Bacterial strains and growth conditions. Bacterial strains and plasmids used in this study are listed in Table 1. Luria-Bertani (LB) broth was used for routine growth of E. amylovora and Escherichia coli strains. An $h r p$-inducing medium (HMM) $-1 \mathrm{~g}$ of $\left(\mathrm{NH}_{4}\right)_{2} \mathrm{SO}_{4}$, $0.246 \mathrm{~g}$ of $\mathrm{MgCl}_{2} \cdot 6 \mathrm{H}_{2} \mathrm{O}, 0.1 \mathrm{~g}$ of $\mathrm{NaCl}, 8.708 \mathrm{~g}$ of $\mathrm{K}_{2} \mathrm{HPO}_{4}$, and $6.804 \mathrm{~g}$ of $\mathrm{KH}_{2} \mathrm{PO}_{4}$-supplemented with $10 \mathrm{mM}$ galactose as carbon source was used for T3SS gene expression, whereas a modified basal medium A (MBMA) - $3 \mathrm{~g}$ of $\mathrm{KH}_{2} \mathrm{PO}_{4}, 7 \mathrm{~g}$ of $\mathrm{K}_{2} \mathrm{HPO}_{4}$, $1 \mathrm{~g}$ of $\left(\mathrm{NH}_{4}\right)_{2} \mathrm{SO}_{4}, 2 \mathrm{ml}$ of glycerol, $0.5 \mathrm{~g}$ of citric acid, and $0.03 \mathrm{~g}$ of $\mathrm{MgSO}_{4}$ - supplemented with $1 \%$ sorbitol was used for amylovoran production (Ancona et al. 2014; Wang et al. 2009). When required, antibiotics were used at the following concentrations: ampicillin at $100 \mu \mathrm{g} \mathrm{ml}-1$, kanamycin $(\mathrm{Km})$ at $50 \mu \mathrm{g} \mathrm{ml}-1$, and chloramphenicol $(\mathrm{Cm})$ at $10 \mu \mathrm{g} \mathrm{ml}^{-1}$. Primer sequences used in this study for mutant construction, mutant confirmation, and quantitative reversetranscription polymerase chain reaction (PCR) are listed in Supplementary Table S1.

Mutant generation by $\boldsymbol{\lambda}$-red recombinase cloning. To generate Erwinia amylovora Ea1189 mutant strains, the $\lambda$-red recombinase cloning method was performed, as described previously (Zhao et al. 2009a). Briefly, competent cells of E. amylovora strain carrying pKD46 were prepared by subculturing in LB with $0.1 \%$ arabinose to exponential phase (optical density at $600 \mathrm{~nm}\left[\mathrm{OD}_{600}\right]=0.8$, approximately $8 \times 10^{8} \mathrm{CFU} / \mathrm{ml}$ ) and washing with cold sterile water. These cells were transformed by electroporation with recombinant DNA fragments, which contain a selection marker $\left(\mathrm{Cm}^{\mathrm{R}}\right.$ or $\left.\mathrm{Km}^{\mathrm{R}}\right)$ flanked with a 50-nucleotide homology from the target gene or region. Plasmids pDK32 or pKD13 were used as a template. Double mutant strains were generated using single mutants as a background. For complementation, the genomic region containing the native promoter and coding sequence of the target gene was PCR amplified and cloned into pWSK29. The resulting plasmids were verified by sequencing at the University of Illinois at Urbana-Champaign Core sequencing facility.

Virulence assay on immature pear. Overnight cultures of E. amylovora wild-type (WT), mutant, and complementation strains in LB were harvested and suspended in $0.5 \times$ phosphatebuffered saline (PBS) to $\mathrm{OD}_{600}=0.1$ and then diluted 100 times (approximately $10^{6} \mathrm{CFU} / \mathrm{ml}$ ) (Zhao et al. 2009b). Immature 'Bartlett' pear fruit (Pyrus communis L.) were surface sterilized with $10 \%$ bleach for $10 \mathrm{~min}$, rinsed with sterile distilled water, and air dried. Pear fruit were pricked with a sterile needle, inoculated with $2 \mu \mathrm{l}$ of bacterial suspension, and incubated at $28^{\circ} \mathrm{C}$ in a humidified chamber in darkness. Disease symptoms were recorded at 4 and 8 days postinoculation (DPI). The experiments were repeated at least twice in triplicate.

Cetylpyrimidinium chloride assay. Amylovoran production was measured using cetylpyrimidinium chloride (CPC), as described previously (Bellemann et al. 1994; Zhao et al. 2009b). Overnight cultures of E. amylovora WT, mutant, and complementation strains in LB were harvested, washed, and inoculated into $5 \mathrm{ml}$ of MBMA medium to $\mathrm{OD}_{600}=0.2$ (approximately $2 \times 10^{8} \mathrm{CFU} / \mathrm{ml}$ ). After $24 \mathrm{~h}$ of incubation at $28^{\circ} \mathrm{C}$ with shaking, $1 \mathrm{ml}$ of each culture was centrifuged at $4,500 \times g$ for $10 \mathrm{~min}$, and $50 \mu \mathrm{l}$ of CPC at $50 \mathrm{mg} / \mathrm{ml}$ was added to the supernatant. After $10 \mathrm{~min}$ of incubation, amylovoran production was quantified by measuring $\mathrm{OD}_{600}$ turbidity and normalized for a cell density of 1.0. Each experiment was performed in triplicate and repeated three times. Statistical analysis was performed using Student's $t$ test, with $P<0.05$ considered to be statistically significant.

Motility assay. Overnight cultures of E. amylovora WT, mutant, and complementation strains in LB were harvested, washed, and suspended in $0.5 \times \mathrm{PBS}$ to $\mathrm{OD}_{600}=1$ (approximately $10^{9} \mathrm{CFU} / \mathrm{ml}$ ). The bacterial suspensions were then plated onto the center of the motility plates (Zhao et al. 2009b) - $10 \mathrm{~g}$ of Bacto tryptone (Becton Dickinson), $5 \mathrm{~g}$ of NaCl, and $2.5 \mathrm{~g}$ of agar (plant tissue culture agar; PhytoTechnology Laboratories) per liter-and incubated at $28^{\circ} \mathrm{C}$. Diameters of movement were measured at 24 and $48 \mathrm{~h}$ postinoculation, and the experiments were repeated three times with three

TABLE 1. Bacterial strains and plasmids used in this study

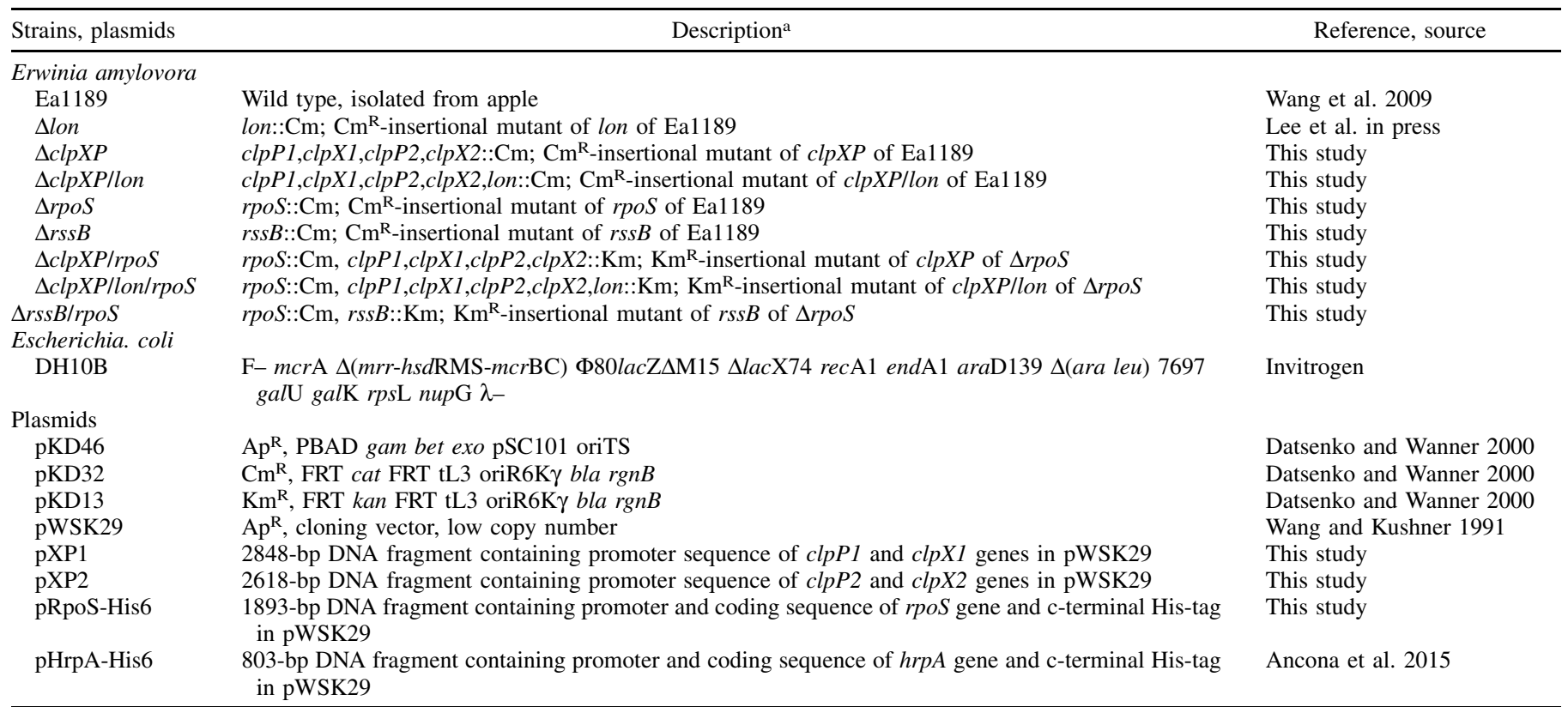

a Abbreviations: $\mathrm{Cm}^{\mathrm{R}}, \mathrm{Km}^{\mathrm{R}}$, and $\mathrm{Ap}^{\mathrm{R}}$ indicate resistant to chloramphenicol, kanamycin, and ampicillin, respectively. 
replicates. Statistical analysis was performed using Student's $t$ test, with $P<0.05$ considered as statistically significant.

Western blot. Western blotting analyses of RpoS and HrpA proteins were performed as previously described (Ancona et al. 2016; Lee et al. in press). Briefly, equal amount of cell lysates from E. amylovora cultures were separated by sodium dodecyl sulfate polyacrylamide gels followed by transfer to polyvinylidene fluoride membrane (Millipore). After blocking with 5\% milk in PBS, membranes were incubated with rabbit anti-His antibodies at $1.0 \mu \mathrm{g} / \mathrm{ml}$ (GeneScript) or rabbit anti-RNA polymerase $\beta$ antibodies (1:2000 dilution; Abcam) and then horseradish peroxidase-linked antirabbit immunoglobulin $\mathrm{G}$ antibodies (1:10,000 dilution; Amersham Bioscience). Protein bands were detected using enhanced chemiluminescence reagents (Pierce) and an ImageQuant LAS 4010 CCD camera (GE Healthcare). This experiment was repeated three times with similar results.

\section{RESULTS}

ClpXP positively contributes to virulence in $E$. amylovora. Based on published genome sequences (Bell et al. 2004; Blattner et al. 1997; Glasner et al. 2011; Smits et al. 2010a,b), E. amylovora and its closely related species E. pyrifoliae contain two sets of clpXP genes, whereas Escherichia coli and two related enterobacterial plant pathogens, Pectobacterium atrosepticum and D. dadantii, contain only one set of $c l p X P$ genes (Fig. 1A). However, the location of the $\operatorname{clpXP}$ genes (i.e., between tig, encoding a chaperone protein, and $(o n)$ is conserved among these bacteria. The deduced amino acids of Erwinia amylovora ClpP1, ClpX1, and ClpX2 share high identities and similarities with their homologs of Escherichia coli, P. atrosepticum and D. dadantii (Table 2), whereas Erwinia amylovora
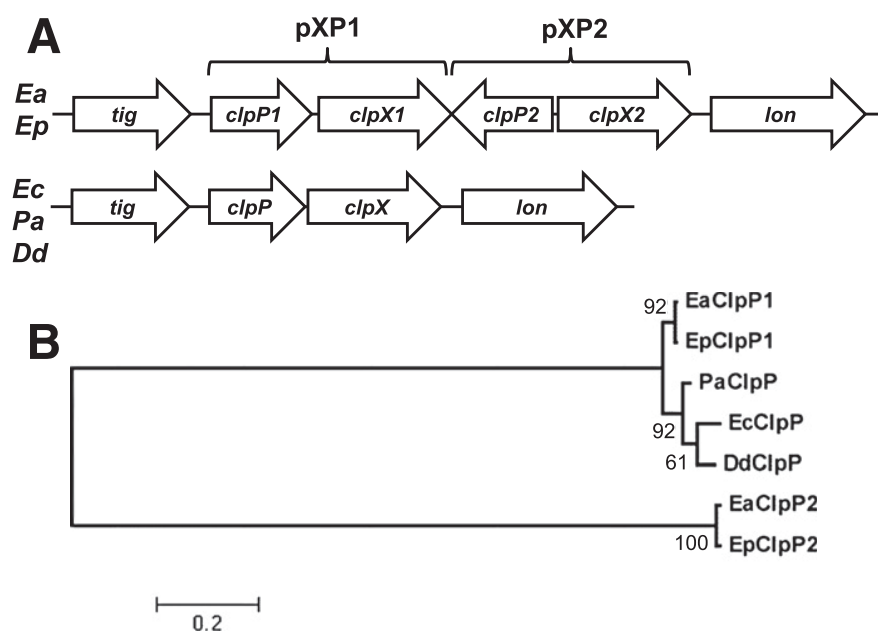

Fig. 1. Comparison of ClpXP in Erwinia amylovora and other related species. A, Schematic maps of the $\operatorname{clp} P$ and $\operatorname{clpX}$ genes. For complementation of the mutant strains, plasmids pXP1, containing $c l p P 1$ and $c l p X 1$, and pXP2, containing clpP2 and $c l p X 2$, were constructed. B, Phylogenetic tree of ClpP proteins. Based on deduced amino acid sequences, a phylogenetic neighbor-joining tree was generated using MEGA 5.0, and bootstrap values were indicated at each node. $\mathrm{Ea}=$ Erwinia amylovora, $\mathrm{Ep}=$ Erwinia pyrifoliae, $\mathrm{Ec}=$ Escherichia coli, $\mathrm{Pa}=$ Pectobacterium atrosepticum, and $\mathrm{Dd}=$ Dickeya dadantii .
$\mathrm{ClpP} 2$ is less conserved in both length and deduced amino acid sequence, and the gene also lies in the opposite direction relative to other $\operatorname{clpXP}$ genes (Fig. 1A). Phylogenetic analysis also separated $\mathrm{ClpP} 2$ of E. amylovora and E. pyrifoliae from other ClpP homologs and clustered into another clade (Fig. 1B).

To determine the role of ClpXP in E. amylovora virulence, a clpXP mutant was generated by deleting all four genes (Fig. 1A). For complementation, each set of $\operatorname{clpXP}$ genes (i.e., $\operatorname{clpXP1}$ and $c l p X P 2)$ was cloned and transformed into the mutant (Fig. 1A). A virulence assay on immature pear fruit showed that the $\operatorname{clpXP}$ mutant caused slightly delayed disease progress as compared with the WT (Fig. 2A), indicating that ClpXP contributes to full virulence in E. amylovora. The complementation strain carrying pXP1 (containing the $c l p X P 1$ genes) but not pXP2 (containing the clpXP2 genes) completely restored disease symptoms, comparable with the WT (Fig. 2A), indicating that the first set of $c l p X P$ genes in E. amylovora is functional under tested conditions.

Our previous studies showed that Lon plays a major role in E. amylovora virulence by negatively regulating T3SS and amylovoran production (Lee et al. in press). In order to determine the effect of lacking both Lon and ClpXP, a clpXP/lon mutant was generated. Interestingly, the mutant exhibited a noticeable reduction of necrotic lesions on immature pear fruit at $8 \mathrm{DPI}$, which could also be rescued by $\mathrm{pXP1}$, but not $\mathrm{pXP} 2$ (Fig. 2B), further suggesting that ClpXP1 are functional under tested conditions and that lack of both ClpXP and Lon leads to noticeable delayed disease progress.

Accumulation of $R p o S$ contributes to delayed disease progress in the $\boldsymbol{c l p X P}$ mutant. RpoS has been regarded as one of the physiologically important ClpXP substrates (Battesti et al. 2011; Flynn et al. 2003). Under favorable growth conditions and at early growth stages, degradation of RpoS by ClpXP with the aid of RssB restricts its activity posttranslationally (Schweder et al. 1996). To determine whether the lack of ClpXP led to RpoS accumulation and thus, reduced virulence, deletion mutants of $r p o S$ in the WT and rss $B, \operatorname{clpXP}$, and $c l p X P / l o n$ mutant backgrounds were generated. Virulence assay showed that the $r p o S, r s s B$, and $r s s B / r p o S$ double mutants were as pathogenic as the WT (Fig. 2C). Interestingly, the clpXP/rpoS mutant exhibited disease progress comparable with the WT, whereas deletion of rpoS in the clpXP/lon mutant did not change the $c l p X P / l o n$ mutant in causing reduction of necrotic lesions at $8 \mathrm{DPI}$ (Fig. 2C).

To verify whether RpoS is accumulated in the $c l p X P$ mutant and contributes to disease progress, the RpoS protein level at different time points in HMM was determined by Western blot. E. amylovora grew slowly at the first $12 \mathrm{~h}$ of incubation (hoi) and reached the stationary phase at 24 hoi in HMM (Supplementary Fig. S1A). The intracellular level of RpoS in E. amylovora was maintained at a low level until 12 hoi and increased starting at 18 hoi, approximately the late exponential or early stationary phase (Fig. 3A). Compared with the WT, RpoS accumulation was approximately 16- and 7-fold higher in the $c l p X P$ and $c l p X P / l o n$ mutants, respectively, and $r s s B$ mutant at 6 hoi (Fig. 3B). These results indicate that RpoS accumulation is growth-dependent and under the control of RssB and $\mathrm{ClpXP}$. These results also suggest that higher accumulation of RpoS in the $\operatorname{clp} X P$ mutant might influence its virulence. However, reduced virulence in the $c l p X P / l o n$ and $c l p X P / l o n / r p o S$ mutants might be caused by unknown factors independent of RpoS.

TABLE 2. Percentage of identity and similarity of deduced amino acid sequences for ClpXP in Erwinia amylovora and related enterobacterial species

\begin{tabular}{|c|c|c|c|c|c|c|c|}
\hline Protein $^{a}$ & Length & Identity & Similarity & Protein $^{a}$ & Length & Identity & Similarity \\
\hline Ea ClpP1 & 207 & $\ldots$ & $\ldots$ & Ea ClpX1 & 424 & $\ldots$ & $\ldots$ \\
\hline Ea ClpP2 & 198 & 36.4 & 68.2 & Ea ClpX2 & 424 & 98.3 & 99.1 \\
\hline Ep ClpP1 & 207 & 99 & 99 & Ep ClpX1 & 424 & 98.8 & 99.8 \\
\hline Ep ClpP2 & 198 & 36.4 & 68.9 & Ep ClpX2 & 424 & 97.2 & 98.6 \\
\hline Ec ClpP & 207 & 87.4 & 96.6 & Ec ClpX & 424 & 91.5 & 97.6 \\
\hline $\mathrm{Pa}$ ClpP & 207 & 88.4 & 96.1 & $\mathrm{~Pa}$ ClpX & 424 & 90.3 & 97.2 \\
\hline Dd ClpP & 207 & 87.9 & 96.6 & Dd ClpX & 424 & 89.2 & 96.5 \\
\hline
\end{tabular}

${ }^{\mathrm{a}} \mathrm{Ea}=$ E. amylovora, $\mathrm{Ep}=$ E. pyrifoliae, $\mathrm{Ec}=$ Escherichia coli, $\mathrm{Pa}=$ Pectobacterium atrosepticum, and Dd = Dickeya dadantii. 
RpoS accumulation leads to reduced expression of the T3SS. In E. amylovora, T3SS is activated at early growth stages in nutrient-limited conditions (Ancona et al. 2015; Wei et al. 2000). Expression of the T3SS genes $h r p L$ and $h r p A$ peaked at 6 hoi in vitro, and then the $h r p L$ gene expression was reduced to basal level, whereas transcription of $h r p A$ was maintained at a relatively high level until 24 hoi. To determine the effect of RpoS accumulation on T3SS in the $r s s B, c l p X P$, and $\operatorname{clpXP/lon}$ mutants, Western blot analysis of HrpA protein was performed (Fig. 3C). The abundance of HrpA was reduced approximately 3-, 12-, and 50-fold in the $r s s$, $c l p X P$, and $c l p X P / l o n$ mutants, respectively, as compared with the WT. Deletion of $r p o S$ in the $r s s B$ and $\operatorname{clpXP}$ mutant backgrounds rescued HrpA expression to levels comparable with those in the rpoS mutant. Deletion of rpoS in the clpXP/lon mutant also increased the HrpA protein level similar to that in the lon mutant, which is approximately twofold higher than that of the WT (Lee et al. in press). These results indicate that RpoS accumulation led to reduced expression of the T3SS.

RpoS suppresses amylovoran production. Previous characterization of the rpoS mutant in E. amylovora strain CFBP 1430 proposed the regulatory role of RpoS in EPS production and motility (Santander et al. 2014); therefore, we determined EPS production in the $\operatorname{clp} X P$ mutant and its related mutant strains. Amylovoran
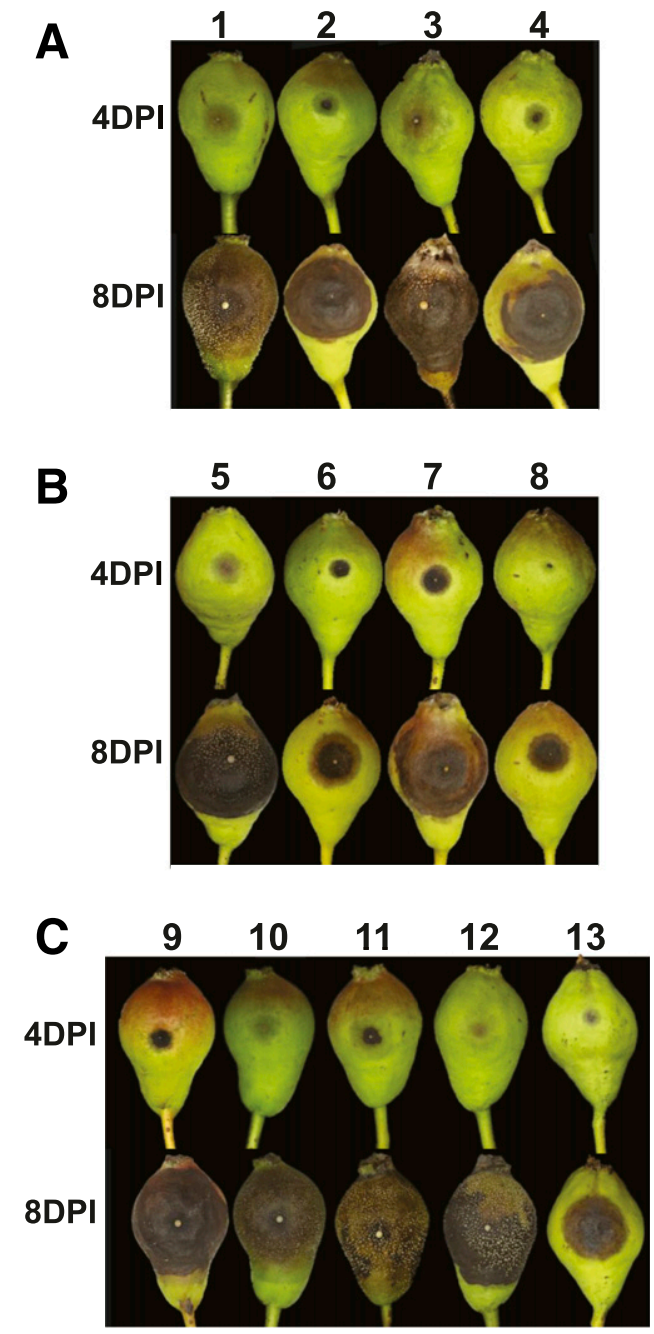

Fig. 2. ClpXP contributes to virulence in Erwinia amylovora, possibly by affecting RpoS accumulation. Disease symptoms caused by the wild-type (WT), mutants, and complementation strains on immature pear fruits at 4 and 8 days postinoculation (DPI). A, Column $1=$ Ea1189 WT, $2=\operatorname{clpXP}, 3=\operatorname{clpXP}$ (pXP1), and $4=$ clpXP (pXP2); B, column $5=$ lon, $6=$ clpXP/lon, $7=$ clpXP/lon (pXP1), and $8=\operatorname{clpXP} /$ lon $(\mathrm{pXP} 2)$; and $\mathbf{C}$, column $9=\operatorname{rpoS}, 10=r s s B, 11=$ rssB/rpoS, $12=$ clpXP/rpoS, and $13=c l p X P / l o n / r p o S$. production was barely detected in the $c l p X P$ mutant but increased in its complementation strain with pXP1, indicating that ClpXP positively affects amylovoran production (Fig. 4A). Consistently, amylovoran production in the $c l p X P / l o n$ mutant was lower than that in the lon mutant (Lee et al. in press) and in the clpXP/lon complementation strain with $\mathrm{pXP} 1$. In contrast, amylovoran production was approximately eightfold higher in the rpoS mutant as compared with that of the WT (Fig. 4B), indicating that RpoS negatively affects amylovoran production. Similar to the $\operatorname{clpXP}$ mutant, the $r s s B$ mutant also exhibited reduced amylovoran production, which could be rescued by deletion of $r p o S$ in both mutant backgrounds. This further confirms that RpoS negatively affects amylovoran

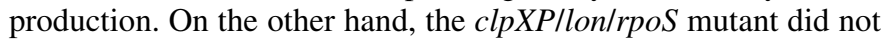
show any significant difference in amylovoran production as compared with the $c l p X P / r p o S$ mutant.

ClpXP is required for motility partially by inhibiting RpoS accumulation. Our recent study reported that the lon mutant is nonmotile mainly due to the accumulation of RcsA/RcsB proteins (Lee et al. in press). Interestingly, the $\operatorname{clp} X P$ mutant also exhibited a nonmotile phenotype, whereas the $\operatorname{clpXP/lon}$ mutant exhibited reduced motility as compared with the WT (Fig. 5A; Supplementary Fig. S2). Complementation of the clpXP/lon mutant with pXP1 returned to the nonmotile phenotype, similar to the $c l p X P$ or lon mutant, whereas the nonmotile phenotype of the $c l p X P$ mutant could not be rescued by pXP1. On the other hand, the rpoS, $r s s B$, and $r p o S / r s s B$ mutants all exhibited reduced motility as compared with the WT (Fig. 5B). Deletion of $r p o S$ rendered the cell motile in the $\operatorname{clpXP}$ mutant, indicating that ClpXP positively affects motility in E. amylovora, possibly by inhibiting RpoS accumulation. However, unexpectedly, the clpXP/lon/rpoS mutant also exhibited the nonmotile phenotype (Fig. 5B). Some of these results seemingly contradicted with each other, suggesting that explaining what controls motility might be difficult at this stage.
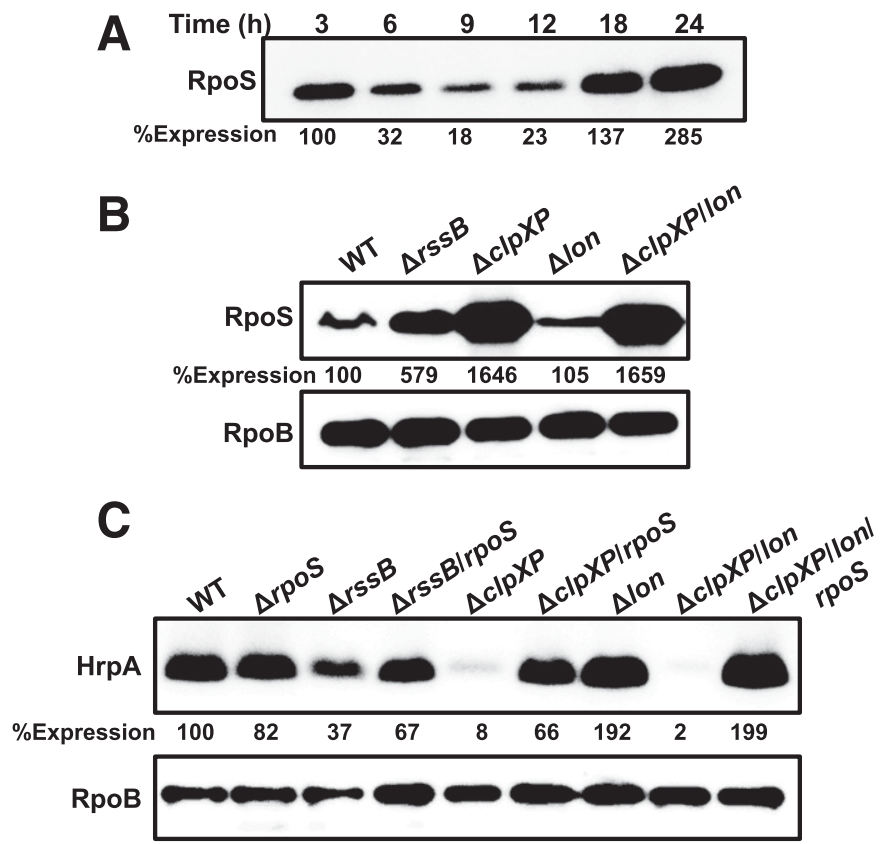

Fig. 3. ClpXP/RssB-dependent RpoS degradation contributes to type III secretion system gene expression. A, Abundance of RpoS-His6 protein in the wild-type (WT) grown in hrp-inducing minimal medium (HMM) at different hours of inoculation at $18^{\circ} \mathrm{C}$. B, Abundance of RpoS-His6 protein in the WT and mutant strains grown in $\mathrm{HMM}$ at $18^{\circ} \mathrm{C}$ for $6 \mathrm{~h}$. C, Abundance of HrpAHis6 protein in the WT and mutant strains grown in $\mathrm{HMM}$ at $18^{\circ} \mathrm{C}$ for $6 \mathrm{~h}$. Abundance of the RpoB protein was used as a loading control, and relative protein abundance (percent expression) was calculated using ImageJ software. These experiments were repeated three times with similar results. 


\section{DISCUSSION}

Posttranslational regulation effectively controls various metabolic enzymes and transcription factors under changing environmental conditions (Sauer and Baker 2011). ClpXP, an intracellular protease belonging to the $\mathrm{AAA}^{+}$family protein, is known to be responsible for regulating more than 50 functional proteins (Flynn et al. 2003). One of its major substrates is RpoS $\sigma$ factor, which affects the expression of genes necessary for growth at stationary phase, stress responses, and virulence (Battesti et al. 2011; Patten et al. 2004). In this study, we showed that ClpXP contributes to E. amylovora virulence by delaying RpoS accumulation. If accumulated at a high level, RpoS suppresses T3SS gene expression, amylovoran production, and motility. In addition, E. amylovora lacking both ClpXP and Lon exhibited reduced virulence independent of RpoS, suggesting that ClpXP or Lon proteases are indispensable to maintain certain functions for E. amylovora virulence.

ClpXP has been shown to activate full T3SS gene expression in several enterobacterial pathogens. In EHEC, mutation in $\operatorname{clpXP}$ caused significantly reduced expression of the enterocyte effacement (LEE)-encoded T3SS genes due to accumulation of GrlR and RpoS (Iyoda and Watanabe 2005). Increased levels of GrlR in the Escherichia coli clpXP mutant directly inhibit the activity of GrlA, which positively regulates the LEE-encoded regulator (Ler), a master

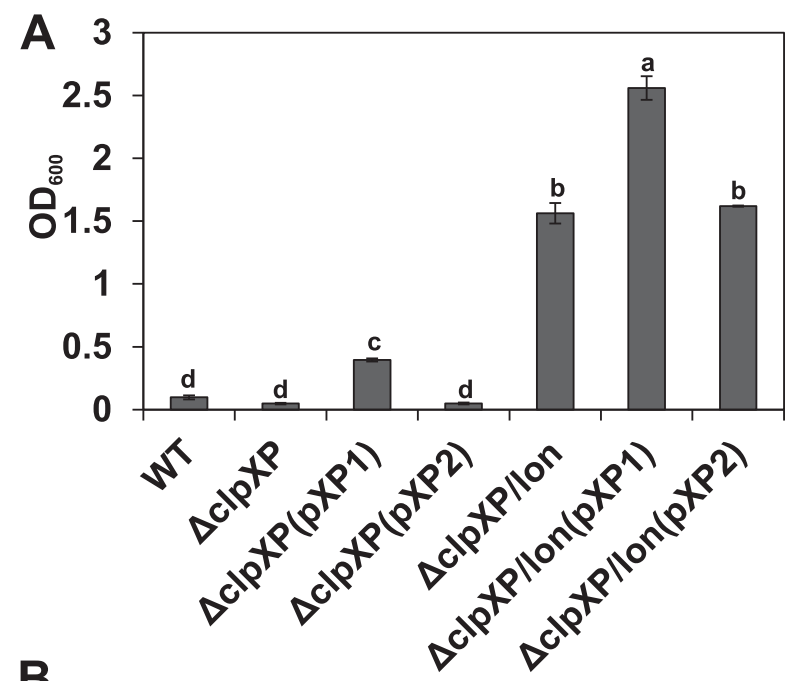

B

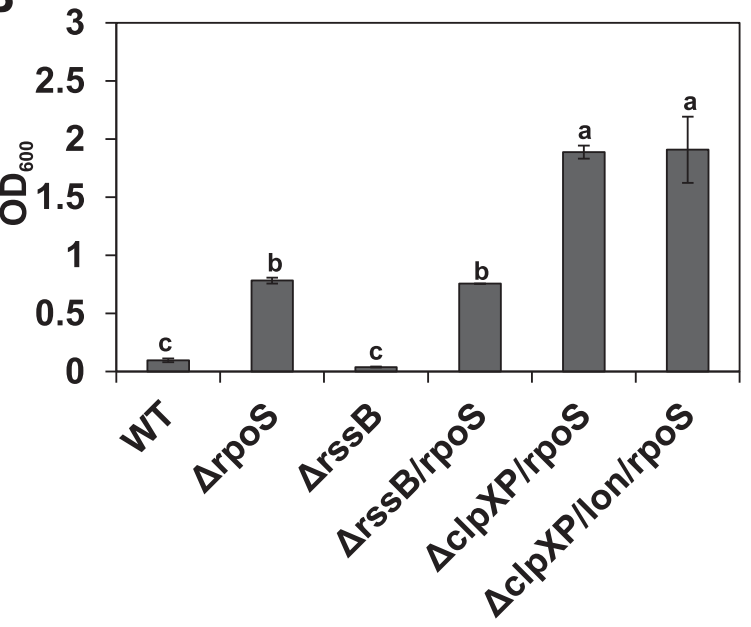

Fig. 4. ClpXP affects amylovoran production by inhibiting RpoS accumulation. A and $\mathbf{B}$, Amylovoran production of the wild-type (WT), mutants, and complementation strains grown in modified basal medium A at $28^{\circ} \mathrm{C}$ for $24 \mathrm{~h}$. Presented values are representative of three independent experiments with similar results. Error bars indicate standard deviation of three replicates. Values marked with the same letter do not differ significantly $(P<0.05)$ regulator of T3SS genes (Deng et al. 2004). Yersinia pestis utilizes both ClpXP and Lon to activate T3SS by degrading a small histone-like protein (YmoA), which represses LcrF, another master regulator of T3SS genes (Hoe and Goguen 1993; Jackson et al. 2004; Lambert de Rouvroit et al. 1992). In the soft rot plant pathogen $D$. dadantii, ClpXP-dependent RpoS degradation has been proposed to positively regulate T3SS by lowering the expression of RNA-binding protein RsmA (repressor of secondary metabolites), a homolog of CsrA (Ancona et al. 2016; Li et al. 2010). Analysis of promoter sequence in E. coli showed that the csrA gene has two RpoS-dependent promoters (Yakhnin et al. 2011). In this study, we also found that excess accumulation of RpoS at an early growth stage suppresses T3SS gene expression. However, in Erwinia amylovora, CsrA positively regulates various virulence traits, including T3SS and amylovoran (Ancona et al. 2016).

Lon, AmyR (amylovoran repressor), and several two-component systems were previously identified as negative regulators of amylovoran production in E. amylovora (Lee et al. in press; Li et al. 2014; Wang et al. 2012; Zhao et al. 2009b). In this study, we provided evidence that RpoS also acts as a negative regulator of amylovoran production, as previously reported (Santander et al. 2014). This is also consistent with previous findings in Escherichia coli that the rpoS mutant overproduces EPS (Ferrieres et al. 2009; Ionescu and Belkin 2009). It has been suggested that EPS overproduction in the rpoS mutant is an adaptive response, because both RpoS and EPS
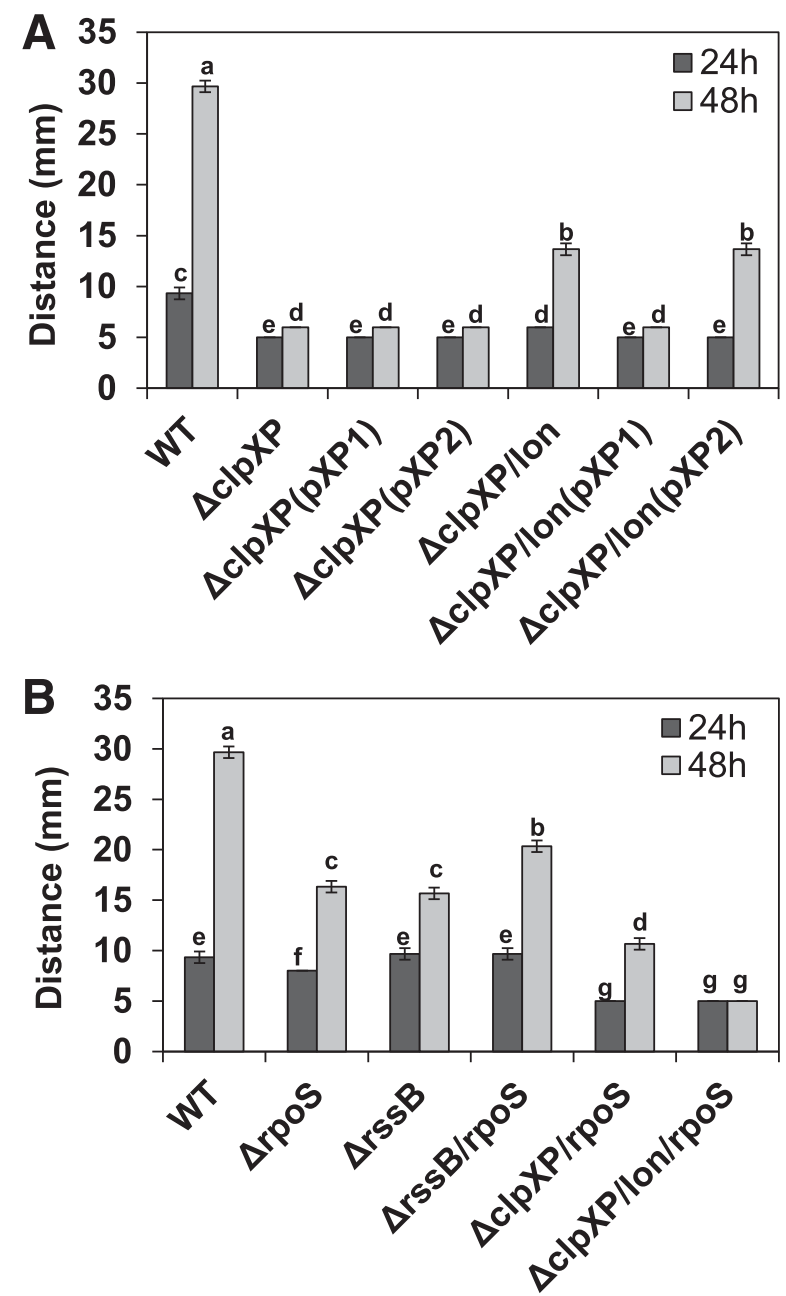

Fig. 5. ClpXP affects motility partially by inhibiting RpoS accumulation. $\mathbf{A}$ and B, The moving distance of the wild-type (WT) and mutant strains on the motility plate. Diameters of the circle around the inoculation site (in millimeters) were measured at 24 and $48 \mathrm{~h}$ of inoculation. Presented values are representative of three independent experiments with similar results. Error bars indicate standard deviation of three replicates. Values marked with the same letter do not differ significantly $(P<0.05)$. 
contribute to cell survival in stress conditions (Battesti et al. 2011; Flemming et al. 2007). Transcriptome analyses in E. coli revealed that the $r p o S$ mutant exhibits increased transcript level of $r c s A$, which encodes RcsA, a rate-limiting factor in EPS production (Dong et al. 2011; Ionescu and Belkin 2009). On the other hand, the Rcs phosphorelay system also activates small RNA RprA to promote translation initiation of $\operatorname{rpoS}$ (Majdalani et al. 2001, 2002), suggesting that RpoS might negatively regulate EPS production through the Rcs phosphorelay system.

In EHEC and Salmonella spp. ClpXP negatively regulates FlhDC, the master regulator of flagellar gene expression, at the posttranslational levels, and the $\operatorname{clpXP}$ mutants are hyperflagellated (Kitagawa et al. 2011; Tomoyasu et al. 2002, 2003). In contrast, the $\operatorname{clpXP}$ mutant of Erwinia amylovora exhibited the nonmotile phenotype, which could be partially rescued by deletion of rpoS. Previously, we have demonstrated that accumulation of $R \operatorname{cs} A / R \operatorname{csB}$ in the lon mutant causes the nonmotile phenotype (Lee et al. in press). In E. amylovora, $\mathrm{Rcs} A / R c s B$ binds to the promoter of $f l h D C$, and its accumulation negatively regulates motility by inhibiting transcription initiation of flhDC. Interestingly, the $c l p X P / l o n$ mutant recovered motility, whereas the $c l p X P / l o n / r p o S$ mutant exhibited the nonmotile phenotype. Because mutation in the rpoS gene in the $c l p X P$ mutant rescued the amylovoran production, the clpXP/lon/rpoS might have increased levels of RcsA/B, which would block motility. However, the effect of lon deletion on motility of the $\operatorname{clpXP}$ mutant remains unexplained.

Taken together, we provided evidence that ClpXP-dependent degradation of RpoS contributes to E. amylovora virulence. As a sixth dissociable subunit of RNAP, $\sigma$ factor plays a critical role in the regulation of transcription initiation in bacteria. $\mathrm{RpoD}(\sigma$ factor 70 ) acts as a primary $\sigma$ factor responsible for the expression of essential genes, whereas alternative $\sigma$ factors such as RpoN and RpoS activate expression of the subset of genes implicated in diverse cellular functions, including virulence and stress responses (Gruber and Gross 2003). The RpoN-HrpL alternative $\sigma$ factor cascade directs the expression of all T3SS genes in E. amylovora (Ancona et al. 2014; McNally et al. 2012; Wei and Beer 1995). Transcription of structural components of the flagella apparatus is also activated by FliA ( $\sigma$ factor 28) (Liu and Matsumura 1995). Because the total level of $\sigma$ factors in the cell exceeds that of core-RNAP, competition between $\sigma$ factors occurs and leads to antagonistic effects on gene expression (Grigorova et al. 2006; Österberg et al. 2011; Piper et al. 2009). RpoS accumulation during the stationary phase downregulates the expression of several genes under the control of RpoD and FliA (Patten et al. 2004; Weber et al. 2005). Mutation in rpoS also upregulates approximately $60 \%$ of RpoN-dependent genes (Dong et al. 2011; Patten et al. 2004). Therefore, it is reasonable to assume that higher levels of RpoS in the $r s s B, c l p X P$, and $\operatorname{clpXP/lon}$ mutants might outcompete other $\sigma$ factors, resulting in overexpression of RpoS regulon and suppression of virulence genes.

In summary, our results showed that ClpXP positively contributes to E. amylovora virulence by maintaining certain levels of RpoS in the cell. However, reduced virulence of the $c l p X P / l o n$ double mutant might be independent of RpoS. Future research should focus on understanding genes under the control of RpoS, ClpXP, and Lon that affect E. amylovora virulence.

\section{ACKNOWLEDGMENTS}

This project was supported by the Agriculture and Food Research Initiative Competitive Grants Program grant number 2016-67013-24812 from the United States Department of Agriculture (USDA) National Institute of Food and Agriculture and USDA Hatch Project ILLU-802-913 (Y. Zhao).

\section{LITERATURE CITED}

Ancona, V., Lee, J. H., Chatnaparat, T., Oh, J., Hong, J. I., and Zhao, Y. 2015. The bacterial alarmone (p)ppGpp activates the type III secretion system in Erwinia amylovora. J. Bacteriol. 197:1433-1443.
Ancona, V., Lee, J. H., and Zhao, Y. 2016. The RNA-binding protein CsrA plays a central role in positively regulating virulence factors in Erwinia amylovora. Sci. Rep. 6: Article 37195. doi:10.1038/srep37195

Ancona, V., Li, W., and Zhao, Y. 2014. Alternative sigma factor RpoN and its modulation protein $\mathrm{YhbH}$ are indispensable for Erwinia amylovora virulence. Mol. Plant Pathol. 15:58-66.

Badger, J. L., and Miller, V. L. 1995. Role of RpoS in survival of Yersinia enterocolitica to a variety of environmental stresses. J. Bacteriol. 177: 5370-5373.

Battesti, A., Majdalani, N., and Gottesman, S. 2011. The RpoS-mediated general stress response in Escherichia coli. Annu. Rev. Microbiol. 65:189-213.

Becker, G., Klauck, E., and Hengge-Aronis, R. 1999. Regulation of RpoS proteolysis in Escherichia coli: The response regulator RssB is a recognition factor that interacts with the turnover element in RpoS. Proc. Natl. Acad. Sci. USA 96:6439-6444.

Bell, K. S., Sebaihia, M., Pritchard, L., Holden, M. T. G., Hyman, L. J., Holeva, M. C., Thomson, N. R., Bentley, S. D., Churcher, L. J. C., Mungall, K., and Atkin, R. 2004. Genome sequence of the enterobacterial phytopathogen Erwinia carotovora subsp. atroseptica and characterization of virulence factors. Proc. Natl. Acad. Sci. USA 101:11105-11110.

Bellemann, P., Bereswill, S., Berger, S., and Geider, K. 1994. Visualization of capsule formation by Erwinia amylovora and assays to determine amylovoran synthesis. Int. J. Biol. Macromol. 16:290-296.

Bernhard, F., Coplin, D. L., and Geider, K. 1993. A gene cluster for amylovoran synthesis in Erwinia amylovora: Characterization and relationship to cps genes in Erwinia stewartii. Mol. Gen. Genet. 239:158-168.

Blattner, F. R., Plunkett, G., Bloch, C. A., Perna, N. T., Burland, V., Riley, M., Collado-Vides, J., Glasner, J. D., Rode, C. K., Mayhew, G. F., and Gregor, J. 1997. The complete genome sequence of Escherichia coli $K-12$. Science 277:1453-1462.

Bougdour, A., Cunning, C., Baptiste, P. J., Elliott, T., and Gottesman, S. 2008. Multiple pathways for regulation of $\sigma^{\mathrm{S}}(\mathrm{RpoS})$ stability in Escherichia coli via the action of multiple anti-adaptors. Mol. Microbiol. 68:298-313.

Bougdour, A., Wickner, S., and Gottesman, S. 2006. Modulating RssB activity: IraP, a novel regulator of $\sigma \mathrm{S}$ stability in Escherichia coli. Genes Dev. 20:884-897.

Bretz, J., Losada, L., Lisboa, K., and Hutcheson, S. W. 2002. Lon protease functions as a negative regulator of type III protein secretion in Pseudomonas syringae. Mol. Microbiol. 45:397-409.

Datsenko, K. A., and Wanner, B. L. 2000. One-step inactivation of chromosomal genes in Escherichia coli K-12 using PCR products. Proc. Natl. Acad. Sci. USA 97:6640-6645.

Deng, W., Puente, J. L., Gruenheid, S., Li, Y., Vallance, B. A., Vázquez, A., Barba, J., Ibarra, J. A., O’Donnell, P., and Ashman, K. 2004. Dissecting virulence: Systematic and functional analyses of a pathogenicity island. Proc. Natl. Acad. Sci. USA 101:3597-3602.

Dong, T., Yu, R., and Schellhorn, H. 2011. Antagonistic regulation of motility and transcriptome expression by RpoN and RpoS in Escherichia coli. Mol. Microbiol. 79:375-386

Fang, F. C., Libby, S. J., Buchmeier, N. A., Loewen, P. C., Switala, J., Harwood, J., and Guiney, D. G. 1992. The alternative sigma factor katF (rpoS) regulates Salmonella virulence. Proc. Natl. Acad. Sci. USA 89: 11978-11982.

Ferrieres, L., Thompson, A., and Clarke, D. J. 2009. Elevated levels of $\sigma \mathrm{S}$ inhibit biofilm formation in Escherichia coli: A role for the Rcs phosphorelay. Microbiology 155:3544-3553.

Flavier, A. B., Schell, M. A., and Denny, T. P. 1998. An RpoS $\left(\sigma^{\mathrm{S}}\right)$ homologue regulates acylhomoserine lactone-dependent autoinduction in Ralstonia solanacearum. Mol. Microbiol. 28:475-486.

Flemming, H. C., Neu, T. R., and Wozniak, D. J. 2007. The EPS matrix: The "house of biofilm cells". J. Bacteriol. 189:7945-7947.

Flynn, J. M., Neher, S. B., Kim, Y. I., Sauer, R. T., and Baker, T. A. 2003. Proteomic discovery of cellular substrates of the ClpXP protease reveals five classes of ClpX-recognition signals. Mol. Cell 11:671-683.

Frees, D., Brøndsted, L., and Ingmer, H. 2013. Bacterial proteases and virulence. Pages 161-192 in: Regulated Proteolysis in Microorganisms. Springer, Dordrecht, The Netherlands.

Glasner, J. D., Yang, C. H., Reverchon, S., Hugouvieux-Cotte-Pattat, N., Condemine, G., Bohin, J. P., Gijsegem, F., Yang, S., Franza, T., Expert, D., and Plunkett, G. 2011. Genome sequence of the plant pathogenic bacterium Dickeya dadantii 3937. J. Bacteriol. 193:2076-2077.

Grigorova, I. L., Phleger, N. J., Mutalik, V. K., and Gross, C. A. 2006. Insights into transcriptional regulation and $\sigma$ competition from an equilibrium model of RNA polymerase binding to DNA. Proc. Natl. Acad. Sci. USA 103:5332-5337.

Gruber, T. M., and Gross, C. A. 2003. Multiple sigma subunits and the partitioning of bacterial transcription space. Annu. Rev. Microbiol. 57:441-466.

Hoe, N. P., and Goguen, J. D. 1993. Temperature sensing in Yersinia pestis: Translation of the LcrF activator protein is thermally regulated. J. Bacteriol. 175:7901-7909. 
Ionescu, M., and Belkin, S. 2009. Overproduction of exopolysaccharides by an Escherichia coli K-12 rpoS mutant in response to osmotic stress. Appl. Environ. Microbiol. 75:483-492.

Iyoda, S., and Watanabe, H. 2005. ClpXP protease controls expression of the type III protein secretion system through regulation of RpoS and GrlR levels in enterohemorrhagic Escherichia coli. J. Bacteriol. 187:4086-4094.

Jackson, M. W., Silva-Herzog, E., and Plano, G. V. 2004. The ATP-dependent ClpXP and Lon proteases regulate expression of the Yersinia pestis type III secretion system via regulated proteolysis of YmoA, a small histone-like protein. Mol. Microbiol. 54:1364-1378.

Khan, M. A., Zhao, Y. F., and Korban, S. S. 2012. Molecular mechanisms of pathogenesis and resistance to the bacterial pathogen Erwinia amylovora, causal agent of fire blight disease in Rosaceae. Plant Mol. Biol. Rep. 30:247-260.

Kitagawa, R., Takaya, A., and Yamamoto, T. 2011. Dual regulatory pathways of flagellar gene expression by ClpXP protease in enterohaemorrhagic Escherichia coli. Microbiology 157:3094-3103.

Koczan, J. M., McGrath, M., Zhao, Y. F., and Sundin, G. W. 2009. The contribution of the exopolysaccharide amylovoran and levan to biofilm formation: Implication in pathogenicity. Phytopathology 99:1237-1244.

Lambert de Rouvroit, C., Sluiters, C., and Cornelis, G. R. 1992. Role of the transcriptional activator, VirF, and temperature in the expression of the pYV plasmid genes of Yersinia enterocolitica. Mol. Microbiol. 6:395-409.

Lee, J. H., Ancona, V., and Zhao, Y. F. Lon protease modulates virulence traits in Erwinia amylovora by directly monitoring major regulators and indirectly through the Rcs and Gac-Csr regulatory systems. Mol. Plant Pathol. In press. doi:10.1111/mpp.12566

Lee, J. H., Sundin, G. W., and Zhao, Y. 2016. Identification of the HrpS binding site in the hrpL promoter and effect of the RpoN binding site of HrpS on the regulation of the type III secretion system in Erwinia amylovora. Mol. Plant Pathol. 17:691-702.

Lee, J. H., and Zhao, Y. 2016. Integration host factor is required for RpoNdependent hrpL gene expression and controls motility by positively regulating rsmB sRNA in Erwinia amylovora. Phytopathology 106:29-36.

Li, W., Ancona, V., and Zhao, Y. 2014. Co-regulation of polysaccharide production, motility, and expression of type III secretion genes by EnvZ/OmpR and GrrS/GrrA systems in Erwinia amylovora. Mol. Genet. Genomics 289:63-75.

Li, Y., Yamazaki, A., Zou, L., Biddle, E., Zeng, Q., Wang, Y., Lin, H., Wang, Q., and Yang, C. H. 2010. ClpXP protease regulates the type III secretion system of Dickeya dadantii 3937 and is essential for the bacterial virulence. Mol. Plant-Microbe Interact. 23:871-878.

Liu, X., and Matsumura, P. 1995. An alternative sigma factor controls transcription of flagellar class-III operons in Escherichia coli: Gene sequence, overproduction, purification and characterization. Gene 164:81-84.

Losada, L. C., and Hutcheson, S. W. 2005. Type III secretion chaperones of Pseudomonas syringae protect effectors from Lon-associated degradation. Mol. Microbiol. 55:941-953.

Majdalani, N., Chen, S., Murrow, J., St John, K., and Gottesman, S. 2001. Regulation of RpoS by a novel small RNA: The characterization of RprA. Mol. Microbiol. 39:1382-1394.

Majdalani, N., Hernandez, D., and Gottesman, S. 2002. Regulation and mode of action of the second small RNA activator of RpoS translation, RprA. Mol. Microbiol. 46:813-826.

McNally, R. R., Toth, I. K., Cock, P. J., Pritchard, L., Hedley, P. E., Morris, J. A., Zhao, Y. F., and Sundin, G. W. 2012. Genetic characterization of the HrpL regulon of the fire blight pathogen Erwinia amylovora reveals novel virulence factors. Mol. Plant Pathol. 13:160-173.

Nimtz, M., Mort, A., Domke, T., Wray, V., Zhang, Y., Qiu, F., Coplin, D., and Geider, K. 1996. Structure of amylovoran, the capsular exopolysaccharide from the fire blight pathogen Erwinia amylovora. Carbohydr. Res. 287:59-76.

Nissinen, R. M., Ytterberg, A. J., Bogdanove, A. J., van Wijk, K., and Beer, S. V. 2007. Analyses of the secretomes of Erwinia amylovora and selected hrp mutants reveal novel type III secreted proteins and an effect of HrpJ on extracellular harpin levels. Mol. Plant Pathol. 8:55-67.

Oh, C. S., and Beer, S. V. 2005. Molecular genetics of Erwinia amylovora involved in the development of fire blight. FEMS Microbiol. Lett. 253:185-192.

Österberg, S., Peso-Santos, T. D., and Shingler, V. 2011. Regulation of alternative sigma factor use. Annu. Rev. Microbiol. 65:37-55.

Patten, C. L., Kirchhof, M. G., Schertzberg, M. R., Morton, R. A., and Schellhorn, H. E. 2004. Microarray analysis of RpoS-mediated gene expression in Escherichia coli K-12. Mol. Genet. Genomics 272:580-591.

Peterson, C. N., Levchenko, I., Rabinowitz, J. D., Baker, T. A., and Silhavy, T. J. 2012. RpoS proteolysis is controlled directly by ATP levels in Escherichia coli. Genes Dev. 26:548-553.
Piper, S. E., Mitchell, J. E., Lee, D. J., and Busby, S. J. 2009. A global view of Escherichia coli Rsd protein and its interactions. Mol. Biosyst. 5:1943-1947.

Pruteanu, M., and Hengge-Aronis, R. 2002. The cellular level of the recognition factor RssB is rate-limiting for $\sigma \mathrm{S}$ proteolysis: Implications for RssB regulation and signal transduction in $\sigma \mathrm{S}$ turnover in Escherichia coli. Mol. Microbiol. 45:1701-1713.

Santander, R. D., Monte-Serrano, M., Rodriguez-Herva, J. J., Lopez-Solanilla, E., Rodriguez-Palenzuela, P., and Biosca, E. G. 2014. Exploring new roles for the $r p o S$ gene in the survival and virulence of the fire blight pathogen Erwinia amylovora. FEMS Microbiol. Ecol. 90:895-907.

Sauer, R. T., and Baker, T. A. 2011. AAA ${ }^{+}$proteases: ATP-fueled machines of protein destruction. Annu. Rev. Biochem. 80:587-612.

Schweder, T., Lee, K. H., Lomovskaya, O., and Matin, A. 1996. Regulation of Escherichia coli starvation sigma factor (sigma s) by ClpXP protease. J. Bacteriol. 178:470-476.

Smits, T. H., Jaenicke, S., Rezzonico, F., Kamber, T., Goesmann, A., Frey, J. E., and Duffy, B. 2010a. Complete genome sequence of the fire blight pathogen Erwinia pyrifoliae DSM $12163 \mathrm{~T}$ and comparative genomic insights into plant pathogenicity. BMC Genomics 11:2.

Smits, T. H., Rezzonico, F., Kamber, T., Blom, J., Goesmann, A., Frey, J. E., and Duffy, B. 2010b. Complete genome sequence of the fire blight pathogen Erwinia amylovora CFBP 1430 and comparison to other Erwinia spp. Mol. Plant-Microbe Interact. 23:384-393.

Solis, R., Bertani, I., Degrassi, G., Devescovi, G., and Venturi, V. 2006. Involvement of quorum sensing and RpoS in rice seedling blight caused by Burkholderia plantarii. FEMS Microbiol. Lett. 259:106-112.

Tomoyasu, T., Ohkishi, T., Ukyo, Y., Tokumitsu, A., Takaya, A., Suzuki, M., Sekiya, K., Matsui, H., Kutsukake, K., and Yamamoto, T. 2002. The ClpXP ATP-dependent protease regulates flagellum synthesis in Salmonella enterica serovar Typhimurium. J. Bacteriol. 184:645-653.

Tomoyasu, T., Takaya, A., Isogai, E., and Yamamoto, T. 2003. Turnover of FlhD and FlhC, master regulator proteins for Salmonella flagellum biogenesis, by the ATP-dependent ClpXP protease. Mol. Microbiol. 48: 443-452.

Wang, D., Korban, S. S., and Zhao, Y. 2009. The Res phosphorelay system is essential for pathogenicity in Erwinia amylovora. Mol. Plant Pathol. 10:277-290.

Wang, R. F., and Kushner, S. R. 1991. Construction of versatile low-copynumber vectors for cloning, sequencing and gene expression in Escherichia coli. Gene 100:195-199.

Wang, D., Qi, M., Calla, B., Korban, S. S., Clough, S. J., Cock, P. J., Sundin, G. W., Toth, I., and Zhao, Y. 2012. Genome-wide identification of genes regulated by the Rcs phosphorelay system in Erwinia amylovora. Mol. Plant-Microbe Interact. 25:6-17.

Weber, H., Polen, T., Heuveling, J., Wendisch, V. F., and Hengge, R. 2005. Genome-wide analysis of the general stress response network in Escherichia coli: $\sigma^{\mathrm{S}}$-Dependent genes, promoters, and sigma factor selectivity. J. Bacteriol. 187:1591-1603.

Wei, Z., Kim, J. F., and Beer, S. V. 2000. Regulation of hrp genes and type III protein secretion in Erwinia amylovora by HrpX/HrpY, a novel twocomponent system, and HrpS. Mol. Plant-Microbe Interact. 13:1251-1262.

Wei, Z. M., and Beer, S. V. 1995. hrpL activates Erwinia amylovora hrp gene transcription and is a member of the ECF subfamily of sigma factors. J. Bacteriol. 177:6201-6210.

Yakhnin, H., Yakhnin, A. V., Baker, C. S., Sineva, E., Berezin, I., Romeo, T., and Babitzke, P. 2011. Complex regulation of the global regulatory gene csrA: CsrA-mediated translational repression, transcription from five promoters by $\mathrm{E}^{70}$ and $\mathrm{E} \sigma^{\mathrm{S}}$, and indirect transcriptional activation by CsrA. Mol. Microbiol. 81:689-704.

Yamamoto, T., Sashinami, H., Takaya, A., Tomoyasu, T., Matsui, H., Kikuchi, Y., Hanawa, T., Kamiya, S., and Nakane, A. 2001. Disruption of the genes for ClpXP protease in Salmonella enterica serovar Typhimurium results in persistent infection in mice, and development of persistence requires endogenous gamma interferon and tumor necrosis factor alpha. Infect. Immun. 69:3164-3174.

Zhao, Y., Sundin, G. W., and Wang, D. 2009a. Construction and analysis of pathogenicity island deletion mutants of Erwinia amylovora. Can. J. Microbiol. 55:457-464.

Zhao, Y., Wang, D., Nakka, S., Sundin, G. W., and Korban, S. S. 2009b. Systems level analysis of two-component signal transduction systems in Erwinia amylovora: Role in virulence, regulation of amylovoran biosynthesis and swarming motility. BMC Genomics 10:245. 